\title{
Is determinism more favorable than indeterminism for the causal Markov condition?
}

\author{
Isabelle Drouet
}

\begin{abstract}
The present text comments on a paper by Daniel Steel, in which the author claims to extent from the deterministic to the general case the result according to which the causal Markov condition is satisfied by systems with jointly independent exogenous variables. I show that Steel's claim cannot be accepted unless one is prepared to abandon standard causal modeling terminology. Correlatively, I argue that the most fruitful aspect of Steel's paper consists in a realist conception of error terms and I show how this conception sheds new light on the relationship between determinism and the causal Markov condition.
\end{abstract}

\section{Introduction}

Despite the stir that Spirtes et al. 1993 or Pearl 2000 caused in the philosophical community, it must be recognized that, in one way or another (see Williamson 2002 for a suggestion to that effect), Bayesian networks can contribute to infer causal knowledge from statistical data. Still they can be used for causal inference purposes only when the causal Markov condition ("CMC" from now onwards) holds. Roughly, this condition states that every phenomenon is probabilistically independent from all its non-effects conditional on its direct causes. Whether, or rather when, it holds remains a debated question.

It is usually mentioned in favor of the $\mathrm{CMC}$ that it is true for deterministic systems with jointly independent exogenous variables. ${ }^{1}$ Reciprocally, counterexamples to the CMC often involve indeterminism. Now, Steel 2005 claims to establish that the determinism clause of the case for the CMC is unnecessary that the CMC is true as soon as exogenous variables are jointly independent. If 
correct, Steel's thesis should constitute an important contribution to the CMC debate, and to the larger debate on the possibility to infer causal knowledge from statistical data.

In the present paper, I ponder the correctness of Steel's claim and the contribution of Steel 2005 to the CMC debate. A general presentation of the CMCdeterminism issue is given in Section 2. Then, Section 3 sets out Steel's argument. Section 4 assesses it, and leads to conclude that Steel's claim cannot be accepted unless standard terminology is abandoned. Nevertheless, Section 5 supports that Steel makes an interesting suggestion as to the representation of causal systems, and shows how this suggestion sheds new light on the relationship between determinism and the CMC.

\section{Determinism and the CMC}

The CMC is the causal version of the more general Markov condition:

Definition 1 (Markov condition) Let $\boldsymbol{V}$ be a finite set of variables, $G$ a directed acyclic over $\boldsymbol{V}$ and $p$ a probability distribution over $\boldsymbol{V}$.

$(G, p)$ satisfies the Markov condition if and only if every variable in $\boldsymbol{V}$ is probabilistically independent from all its non-descendants in $G$ conditional on its parents in $G$.

Let us now consider a "system" - that is, in the present context, any group of causally interrelated phenomena. A simple example introduced by Halpern and Pearl $(2005,848)$ consists in a forest that can catch fire because of either lightning or a match lit by an arsonist. This system can be represented by:

1. the variables $L, A, F$ respectively representing whether lightning strikes, whether an arsonist lights a match in the forest, and whether there is a forest fire. ${ }^{2}$ More precisely, $L$ is the binary variable which takes the value 1 if and only if lightning strikes and 0 otherwise, and so on for $A$ and $F$;

2. the directed graph<smiles>[3H][Te][3H]</smiles>

representing the direct causal relationships among the variables $L, A$ and $F$. This graph is "the causal graph" over $\{L, A, F\}$ - or, equivalently, the causal graph for the system under consideration;

3. the probability distribution over $\{L, A, F\}$.

More generally, any system can be represented by a set of variables and the causal graph and probability distribution over this set. Correlatively, the causal version of the Markov condition can be defined as a property of systems:

Definition 2 (Causal Markov condition) Let $S$ be a system represented by the set of variables $\boldsymbol{V}$, the acyclic causal graph $C G$ and the probability distribution $p$ over $\boldsymbol{V}$.

$S$ satisfies the causal Markov condition if and only if $(C G, p)$ satisfies the Markov condition. 
Let me introduce some vocabulary before I set out the usual result concerning the $\mathrm{CMC}$ and determinism:

- a system $S$ whose relevant observable aspects are represented by the variables in $\mathbf{V}$ is:

- acyclic if and only if $C G$ is acyclic;

- deterministic if and only if the values of the $\mathbf{V}$ variables with direct causes in $\mathbf{V}$ are functionally determined by the values of these direct causes;

- the exogenous variables of $S$ are the variables in $\mathbf{V}$ not having direct causes in $\mathbf{V}$;

- the variables in $\mathbf{V}$ are jointly independent if any two non-empty distinct subsets of $\mathbf{V}$ are probabilistically independent.

Now the classic result beyond which Steel pretends to go can be stated:

Theorem 1 (Classic result) Acyclic deterministic systems with jointly independent exogenous variables satisfy the $C M C$.

Here is a proof for Theorem 1:

Let $S$ be an acyclic deterministic system represented by $\{\mathbf{V}, G, p\}$. Let us assume that the exogenous variables in $\mathbf{V}$ are jointly independent.

$S$ satisfies the CMC if and only if any variable in $\mathbf{V}$ is independent for $p$ of its non-descendants in $G$ conditional on its parents in $G$.

Then, let us consider any variable $V$ in $\mathbf{V}$ and show that it is indeed independent of its non-descendants in $G$ conditional on its parents in $G$.

- if $V$ is endogenous, then its value is functionally determined by the values of its parents in $G$. Therefore it is independent of any of its non-descendants in $G$ when one conditionalizes on the set of its parents in $G$;

- if $V_{1}$ is exogenous, the set of its parents in $G$ is empty. Therefore one must simply show that $V$ is (unconditionally) independent of any of its non-descendants in $G$.

Let $V_{2}$ be such a variable.

- if $V_{2}$ is also exogenous, the independence of $V_{1}$ and $V_{2}$ stems from the hypothesis of joint independence of exogenous variables;

- if $V_{2}$ is endogenous, by the determinism hypothesis, its value is determined by the value of the set of its direct causes in $\mathbf{V}$. Beyond, and by acyclicity, this value is determined by that of a set of exogenous variables of $S$, say $\mathbf{W}$. $V_{1}$ does not belong to $\mathbf{W}$ since $V_{2}$ is not a descendant of $V_{1}$. Then the joint independence hypothesis entails that $V_{1}$ is independent from $\mathbf{W}$ and therefore from $V_{2}$.

As a consequence, any variable in $\mathbf{V}$ is independent of its non-descendants in $G$ conditional on its parents in $G: S$ satisfies the CMC. 


\section{Steel's argument}

What is claimed in Steel 2005 is that the determinism clause in Theorem 1 is superfluous. In other words, a system would satisfy the CMC as soon as its exogenous variables are jointly independent. The present section sets out Steel's argument in favor of this claim.

Steel's argument is stated in the framework of "causal functional models" (CFMs for short). Let me explain what they are by first introducing "functional models" (FMs). A FM is defined over a couple of sets of variables, say $\mathbf{X}=$ $\left\{X_{1}, X_{2}, \ldots, X_{n}\right\}$ and $\mathbf{U}=\left\{U_{1}, U_{2}, \ldots, U_{k}\right\}$. A functional model over $(\mathbf{X}, \mathbf{U})$ is $(\mathbf{E}, p)$ where:

- $\mathbf{E}$ is a set of $n$ equations such that each $X_{i}$ appears as a function $f_{i}$ of a non-empty subset of $\left((\mathbf{X} \cup \mathbf{U}) \backslash\left\{X_{i}\right\}\right)$;

- $p$ is a probability distribution over $\mathbf{U}$.

An example of an FM over $\left(\left\{X_{1}, X_{2}, X_{3}\right\},\left\{U_{1}, U_{2}\right\}\right)$ is composed by the equations:

$$
\begin{gathered}
X_{1}=f_{1}\left(U_{1}, U_{2}\right) \\
X_{2}=f_{2}\left(X_{1}, U_{2}\right) \\
X_{3}=f_{3}\left(X_{2}\right)
\end{gathered}
$$

together with a probability distribution $p$ over $\left\{U_{1}, U_{2}\right\} \cdot{ }^{3}$ Now Steel $(2005,9)$ describes $(\mathbf{E}, p)$ "causal" if 1 ) all the equations in $\mathbf{E}$ are causal generalizations and 2) any $X_{i}$ in $\mathbf{X}$ is such that the set DirectCauses $\left(X_{i}\right)$ of its direct causes in $(\mathbf{X} \cup \mathbf{U})$ is included in (but not necessarily equal to) the set of its functional parents in $(\mathbf{X} \cup \mathbf{U})$.

Three remarks must be made before I can actually come to the result established by Steel:

- as noticed by Steel himself $(2005,7)$, there is a "straightforward correspondence between functional models and directed graphs": for an FM $M=(\mathbf{E}, p)$ over $(\mathbf{X}, \mathbf{U})$, there is a unique directed graph $G_{M}$ over $(\mathbf{X} \cup \mathbf{U})$ such that graphical parents in $G_{M}$ exactly correspond to functional parents in E. For our example FM, the corresponding directed graph is as follows:

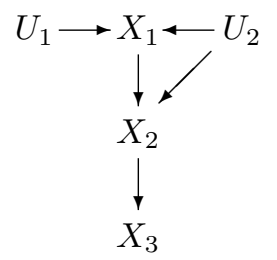

If $G_{M}$ is acyclic (as is the case here), $M$ as well as systems represented by $M$ will themselves be labeled "acyclic";

- if $M$ is a causal functional model, then $G_{M}$ is an over-graph of the causal graph $C G_{M}$ over $\mathbf{D C}_{M}=\bigcup_{X_{i} \in \mathbf{X}}$ DirectCauses $\left(X_{i}\right)$. As will be clearer soon, $C G_{M}$ is the causal graph for systems represented by $M$; 
- if $G_{M}$ is acyclic, then the equations in $\mathbf{E}$ can be restated in such a way that $\mathbf{X}$ variables are functions only of the $\mathbf{U}$ variables from which they descend (Steel 2005, 8). As a consequence, $p$ univocally extends to a probability distribution $p^{\prime}$ over $(\mathbf{U} \cup \mathbf{X})$, which itself univocally restricts to $p^{\prime \prime}$ over $\mathrm{DC}_{M}$.

In terms of CFMs and following the notations already introduced, the result established by Steel is the following:

Theorem 2 (Steel's result) Let $M=(\boldsymbol{E}, p)$ be a $C F M$ over $(\boldsymbol{X}, \boldsymbol{U})$. If $G_{M}$ is acyclic and variables in $\boldsymbol{U}$ are jointly independent for $p$, then $\left(C G_{M}, p^{\prime \prime}\right)$ satisfies the Markov condition.

In terms of systems ${ }^{4}$, it can be formulated as:

Theorem 3 Let $S$ be a system represented by the CFM $M=(\boldsymbol{E}, p)$ over $(\boldsymbol{X}, \boldsymbol{U})$. If $S$ is acyclic and the variables in $\boldsymbol{U}$ are jointly independent for $p$, then $S$ satisfies the $C M C$.

A proof which differs slightly from the one given by Steel but makes it particularly clear what the rationale for the result is, is made up of:

1. a proof of the fact that $\left(G_{M}, p^{\prime}\right)$ satisfies the Markov condition - in other words: a proof of the fact that $\left(G_{M}, p^{\prime}\right)$ is a Bayesian network. This proof runs exactly as the proof that was given for Theorem 1 but with the role of the determinism clause played by functional determination;

2. a derivation of the probabilistic conditional independencies that are required in order for $\left(C G_{M}, p^{\prime \prime}\right)$ to satisfy the Markov condition. Those independencies are established by $d$-separation in the Bayesian network $\left(G_{M}, p^{\prime}\right)$.

The relationship between Steel's result and the determinism / indeterminism - CMC issue consists exactly in the following: CFMs can represent indeterministic as well as deterministic systems. In order to make it clear, Steel gives the following example:

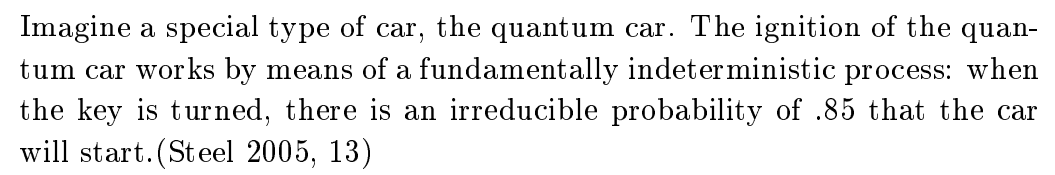

Then Steel $(2005,13)$ explains that the system constituted by the "quantum car" can be represented by a CFM $M$ over $\left(\left\{X_{1}, X_{2}\right\},\left\{U_{1}, U_{2}\right\}\right)$, where

- $X_{1}$ is "a binary variable indicating whether the key is turned $\left(X_{1}=1\right.$ indicates that it has been)";

- $X_{2}$ is "a binary variable representing whether the car starts $\left(X_{2}=1\right.$ indicates that it does)";

- (as far as I understand Steel's treatment of the example) $U_{1}$ represents the reasons why the car may be started;

- $U_{2}$ is a binary variable whose possible values are 0 and 1 and which represents whether the car starts once the key has been turned. 
$M$ is composed of the equations

$$
\begin{aligned}
& X_{1}=f_{1}\left(U_{1}\right) \\
& X_{2}=U_{2} \cdot X_{1}
\end{aligned}
$$

together with a probability distribution $p$ over $\left\{U_{1}, U_{2}\right\}$ which is such that $p\left(U_{2}=1\right)=.85$. With $p$ defined in this way, it becomes clear that $U_{2}$ represents the probabilistic nature of the action of $X_{1}$ on $X_{2}$. More generally, the example makes it clear how any indeterministic system can be represented by a causal functional model, with $\mathbf{U}$ variables representing the probabilistic nature of the action of indeterministic causes on their effects. Consequently, Steel considers that Theorem 2 implies that the CMC is true for any acyclic system with jointly independent exogenous variables, be it deterministic or not. This is what I bring into question. More precisely, I question neither the truth of theorem 2, nor the validity of Steel's proof, but rather the way Steel interprets it.

\section{Assessment of Steel's argument}

Obviously, a necessary condition for Steel's result being interpretable in terms of any acyclic system with jointly independent exogenous variables satisfying the CMC is that the $\mathbf{U}$ variables of a CFM are the exogenous variables of systems represented by that CFM. This is assumed by Steel. Yet he has an hesitation when introducing them, referring to "a set of exogenous variables or error terms" (Steel 2005, 5). It seems to me that this hesitation is meaningful. But this can be justified only after I have told the standard story about exogenous variables, error terms, and the way they differ.

The standard definition of exogenous variables was introduced in Section 2. One has 0 ) a system $S, 1)$ a set $\mathbf{V}=\left\{V_{1}, V_{2}, \ldots V_{n}\right\}$ of variables representing its relevant observable aspects, 2) a graph $C G$ representing the direct causal relations on $\mathbf{V}$. The exogenous variables of $S$ are those variables in $\mathbf{V}$ that do not have any parents in $C G$. All this has already been stated. But from there on one can go one step further in the representation of $S$ and consider a set of $n$ equations such that each $V_{i}$ constitutes the left-hand-side of exactly one equation and is a function of its parents in $C G$ plus one variable $T_{i}$. This set of equations together with the probability distribution over $\mathbf{T}$ constitutes a representation of the system under consideration which is common in the field of causal modeling - see for instance Pearl's "causal models" (Pearl 2000, 27). Therefore this pattern of representation will be referred to as that of "standard causal functional models" ("standard CFMs" for short). The $\mathbf{T}$ variables of standard CFMs have a precise function: they enable a functional representation of the relations between $\mathbf{V}$ variables. Indeed, each $T_{i}$ represents everything that contributes to the determination of the value of $V_{i}$ and yet is not represented by the variables in $\mathbf{V} \backslash\left\{V_{i}\right\}$. More precisely, and following Cartwright's analysis, $\mathbf{T}$ variables "represent, in one heap, measurement errors, omitted factors, and whatever truly probabilistic element there may be in the determination of an effect by its causes" (Cartwright 1999, 9). T variables are those usually called "error terms".

This was the standard story. Let us now come back to Steel's one. Are his U variables "exogenous variables or error terms" (Steel 2005, 5)? Stated in other 
words: what do Steel's U variables represent? It is clear from Steel's paper that $\mathbf{U}$ variables can represent two very different kinds of things:

- some of them, like $U_{1}$ in the quantum car example, represent relevant observable aspects of the system under consideration that happen not to have direct causes in the system. Those variables are exogenous variables in the standard sense of the term;

- the other ones, like $U_{2}$, represent the way probabilistic causes act on their effects. Those variables are not exogenous variables since they do not represent observable relevant aspects of the system under consideration. Moreover, they represent one of the three kinds of influences that error terms in the standard sense represent. But they represent only one of the three kinds of influences that are represented "in one heap" by error terms. As a consequence, they are not error terms standardly speaking.

This analysis easily accounts for the hesitation of Steel when introducing $\mathbf{U}$ : $\mathbf{U}$ is composite object whose elements either are exogenous variables in the standard sense, or represent part of what standard error terms represent. Consequently, U cannot be called "the set of exogenous variables" if one conforms to standard terminology. Stated in another way, Steel's result can be interpreted in terms of truth of the CMC for any acyclic system with jointly independent exogenous variables only if one abandons the standard pattern of functional representation of systems and the sense "exogenous variables" has in this context. Therefore it cannot be said without qualification that Steel established the superfluity of the determinism clause in Theorem 1. Omitting qualification, as Steel does, is seriously misleading.

It could be that all this does not matter much. This would be the case in particular if Steel showed the way towards a proof of the truth of the CMC for any system with jointly independent exogenous variables in the standard sense). More likely, it may be that this can be proved in a way similar to the proof proposed for Steel's result. This seems all the more likely since the given proof-sketch is modular and has already been used to produce a proof of a result which is quite different from Theorem 2 (Pearl 2000, 30). Yet a quick examination of the sketch reveals that one can obtain a final result in terms of joint independence of exogenous variables (standardly defined) only if one adopts a pattern of representation from which error terms are absent. But we saw that the first part of the proof relies in an essential way on the functional determination of the values of the effects by those of their direct causes. In the absence of error terms, such functional determination is exactly equivalent to determinism. In other words, the proof-sketch is no use for one who wants to establish that all acyclic systems with jointly independent exogenous variables satisfy the CMC.

Of course this does not imply that the claim is false. What implies it by contrast is the fact that, under standard terminology, it remains possible for an acyclic system with jointly independent exogenous variables to fail to satisfy the $\mathrm{CMC}$ - provided it is indeterministic. This is the case of Nancy Cartwright's classic example:

Cheap-but-Dirty employs a genuinely probabilistic process to produce the chemical [a chemical that is consumed in a given sewage plant]. The 
probability of getting the desired chemical on any day the factory operates is eighty percent. [...] [Moreover] pollutants are emitted as a by-product whenever the chemical is produced.(Cartwright 1999, 7)

Relevant observable aspects of the systems are represented by three binary variables with value 0 or 1: $\mathrm{O}$ indicating whether Cheap-but-Dirty operates, $\mathrm{S}$ indicating whether sewage is produced, and $\mathrm{P}$ indicating whether pollutant is produced. It is easily seen that only one of them is exogenous in the standard sense: O. Hence, trivially, exogenous variables are jointly independent. Moreover, the system is clearly acyclic. And yet the system does not satisfy the CMC since $\mathrm{O}$ does not screen off $\mathrm{P}$ from $\mathrm{S}$ :

$p(P=1, S=1 \mid O=1)=.8$, whereas

$p(P=1 \mid O=1) \times p(S=1 \mid O=1)=.8 \times .8=.64$.

As a consequence, the result Steel claims to have established remains false under standard terminology.

Acyclicity and joint independence of exogenous variables are sufficient for the CMC only if one accepts as exogenous variables, variables that represent the way probabilistic causes act on their effects. It could be argued that there is nothing wrong with this and, quite the opposite, that this modeling proposition constitutes the very innovation in Steel's paper. This is precisely the position adopted by Steel himself: "The basic insight is that exogenous variables in an FM can be interpreted either as representing causes or genuine indeterminism" (Steel 2005,4 ). The matter is that variables representing the way probabilistic causes act on their effects already existed before Steel's paper, and that they were never called "exogenous variables". Conversely, "exogenous variables" and "systems with jointly independent exogenous variables" already had a meaning before Steel's paper, and this meaning is different from the one they have in Steel 2005. Moreover, Steel does not give any independent justification for substituting his interpretation of exogenous variables to the usual one. Then, his modeling innovation should not be accepted unless the associated terminology were carefully distinguished from the usual one and the author were careful not to claim to enter a pre-existing debate - two things that Steel fails to do.

\section{What Steel's paper suggests}

I would like to end with more positive considerations. Indeed I think that Steel's paper actually contributes to the CMC debate, in spite of the difficulties hitherto highlighted. As the difficulties, the contribution lies in Steel's U variables - and, to be more precise, in those $\mathbf{U}$ variables that are neither exogenous variables nor error terms in the standard sense of these terms. I have already stated that those variables represent only one of the three kinds of influences that standard error terms represent "in one heap", and this appeared to be problematic. But there is another way of looking at this: while standard error terms clearly fail to represent any real entity, Steel's U variables lean towards realism in the use of error terms - by which is meant that error terms are used in such a way that the structure of a causal model matches the real causal structure it represents. More specifically, Steel's U variables suggest to disjoin the influences that are represented "in one heap" by standard error terms, and to represent distinct influences by distinct error terms. 
Following this suggestion, to each variable $V$ representing a relevant observable aspect of the system under consideration would correspond:

- an error term representing the forgotten direct causes of what $V$ represents;

- an error term representing the possible errors in the measurement of $V$;

- for each of $V$ 's probabilistic direct causes, an error term representing the way it acts on $V$.

These non-standard error terms lead to define non-standard causal functional models. Given a system $S$ whose relevant observable aspects are represented by $\mathbf{V}=\left\{V_{1}, V_{2}, \ldots, V_{n}\right\}$, a non-standard CFM for $S$ is $(\mathbf{E}, p)$ where:

- $\mathbf{E}$ is a set of $n$ equations such that each $V_{i}$ is the left-hand-side of exactly one equation and appears as a function of 1 ) its direct causes in $\mathbf{V}$ and 2 ) non-standard error terms $T_{i, 1}, T_{i, 2}, \ldots, T_{i, m}$. Observe that $m$ depends on which variable $V_{i}$ is considered;

- $p$ is the probability distribution over the set $\mathbf{T}$ of non-standard error terms.

Non-standard CFMs complete Steel's CFMs so as to ensure that everything standard CFMs represent is actually represented. This is enough for nonstandard CFMs not implying the important redefinition of usual terminology that Steel's CFMs did convey, and that was identified as problematic. But as for the rest, the two patterns of representation are largely similar. First and foremost, non-standard CFMs allows a characterization of determinism that relies on the very intuition conveyed by Steel's "Definition of Deterministic Functional Models" (Steel 2005, 9):

Definition 3 (Characterization of Determinism) A system is deterministic if it is represented by a non-standard causal functional model with no error term representing the way a probabilistic cause acts on one of its effects.

Then, as Steel's CFMs, non-standard CFMs are in "straightforward correspondence" (Steel 2005, 7) with directed graphs. Accordingly, they and the systems they represent will also be labeled "acyclic" when the corresponding directed graph is acyclic. Moreover, acyclicity of the non-standard CFM $M=(\mathbf{E}, p)$ over $(\mathbf{V}, \mathbf{T})$ remains sufficient for the probability distribution $p$ over $\mathbf{T}$ to univocally extend to $p^{\prime}$ over $(\mathbf{T} \cup \mathbf{V})$. Finally, and still as with Steel's CFMs, the graph $G_{M}$ corresponding to $M$ is an over-graph of the causal graph for systems represented by $M$. Notice that this causal graph is now univocally determined by $M$ (as the directed graph representing the direct causal relations amongst $\mathbf{V}$ variables that are depicted by $\mathbf{E}$ ); therefore it can and will be noted: " $C G_{M}$ ".

Does this tell anything new about the relationship between determinism and the CMC? Here, one may first notice that, under the notations that have just been introduced, the following result holds:

Theorem 4 Let $M=(\boldsymbol{E}, p)$ be an acyclic non-standard $C F M$ over $(\boldsymbol{V}, \boldsymbol{T})$ and $p^{\prime \prime}$ the restriction of $p^{\prime}$ to $\boldsymbol{V}$.

If variables in $\boldsymbol{T}$ are jointly independent, then $\left(C G_{M}, p^{\prime \prime}\right)$ satisfies the Markov condition. 
Theorem 4 is the equivalent, in the framework of non-standard CFMs, of Steel's result. It holds for exactly the same reasons and can also be stated in terms of systems:

Theorem 5 Let $S$ be a system represented by the non-standard CFM $M$ over $(\boldsymbol{V}, \boldsymbol{T})$.

If $M$ is acyclic and variables in $\boldsymbol{T}$ are jointly independent, then $S$ satisfies the $C M C$.

By way of illustration, let us consider a classic (non quantum) car, by which is meant a car that starts each time the key is turned and there is petrol in the tank. Let $S$ be this system. Three variables are needed in order to represent $S: V_{1}$ representing whether the key is turned, $V_{2}$ representing whether there is petrol in the car and $V_{3}$ representing whether the car starts. Let $M=(\mathbf{E}, p)$ be the non-standard CFM representing $S$. Equations in $\mathbf{E}$ are as follows:

$$
\begin{gathered}
V_{1}=g_{1}\left(T_{1,1}, T_{1,2}\right) \\
V_{2}=g_{2}\left(T_{2,1}, T_{2,2}\right) \\
V_{3}=g_{3}\left(Y_{1}, Y_{2}, T_{3,1}, T_{3,2}\right)
\end{gathered}
$$

with $T_{1,1}$ representing the omitted causes of $V_{1}, T_{1,2}$ the possible errors in the measurement of the value of $V_{1}$, and correspondingly for $V_{2}$ and $V_{3}$. Given acyclicity of $S$, Theorem 5 states that a sufficient condition for it to satisfy the $\mathrm{CMC}$ is that the variables in $\left\{T_{1,1}, T_{1,2}, T_{2,1}, T_{2,2}, T_{3,1}, T_{3,2}\right\}$ are jointly independent.

Now, this condition can be refined. Indeed, and as already stressed by Cartwright $(2001,18)$ in another context, what is needed for a proof in the style of Pearl's one to be possible is only the joint independence of the "net effects" of error terms. In our example, "net effects" of error terms correspond to $\left\{T_{1,1}, T_{1,2}\right\},\left\{T_{2,1}, T_{2,2}\right\}$ and $\left\{T_{3,1}, T_{3,2}\right\}$. Then, following Cartwright, it is sufficient that any way of combining them into two non-empty sets is in two independent sets of variables. To be explicit, it is sufficient that:

(1) $\left\{T_{1,1}, T_{1,2}\right\},\left\{T_{2,1}, T_{2,2}\right\}$ and $\left\{T_{3,1}, T_{3,2}\right\}$ are pairwise independent;

(2) $\left\{T_{1,1}, T_{1,2}\right\}$ is independent from $\left\{T_{2,1}, T_{2,2}, T_{3,1}, T_{3,2}\right\}$;

(3) $\left\{T_{2,1}, T_{2,2}\right\}$ is independent from $\left\{T_{1,1} ; T_{1,2}, T_{3,1}, T_{3,2}\right\}$ and

(4) $\left\{T_{3,1}, T_{3,2}\right\}$ is independent from $\left\{T_{1,1}, T_{1,2}, T_{2,1}, T_{2,2}\right\}$.

Joint independence of $\left\{T_{1,1}, T_{1,2}, T_{2,1}, T_{2,2}, T_{3,1}, T_{3,2}\right\}$ is too strong condition for a proof following the sketch given above to be available. In particular it is not necessary for $T_{1,1}$ to be independent from $T_{1,2}$, for $T_{2,1}$ to be independent from $T_{2,2}$, or for $T_{3,1}$ to be independent from $T_{3,2}$.

Stated in terms of a non-standard CFM $M$ over $(\mathbf{V}, \mathbf{T})$, the proof only requires the following: for any two distinct non-empty $\mathbf{V}^{\prime}, \mathbf{V}^{\prime \prime} \subset \mathbf{V}$, the sets of error terms respectively corresponding to the variables in $\mathbf{V}^{\prime}$ and to the variables in $\mathbf{V}^{\prime \prime}$ are independent. More rigorously, and with $\varphi(i)$ the number of error terms in $M$ for $V_{i} \in \mathbf{V}$, the result is as follows:

Theorem 6 Let $M=(\boldsymbol{E}, p)$ be an acyclic non-standard CFM over $\boldsymbol{V}$. If for any two $\boldsymbol{V}^{\prime}=\left\{V_{i}, \ldots, V_{j}\right\}$ and $\boldsymbol{V}^{\prime \prime}=\left\{V_{k}, \ldots, V_{l}\right\}$ non empty disjoint 
subsets of $\boldsymbol{V},\left\{T_{i, 1}, \ldots, T_{i, \varphi(i)}, \ldots, T_{j, 1}, \ldots, T_{j, \varphi(j)}\right\}$ is independent from $\left\{T_{k, 1}, \ldots, T_{k, \varphi(k)}, \ldots, T_{l, 1}, \ldots, T_{l, \varphi(l)}\right\}$, then $\boldsymbol{V}$ satisfies the $C M C$.

For simplicity's sake, let me refer to the antecedent of the implication stated by Theorem 6 as: "V satisfies JI". Now, in terms of systems represented by non-standard CFMs, we have:

Theorem 7 Let $S$ be a system represented by the non-standard CFM $M$. If $M$ is acyclic and satisfies $\boldsymbol{J I}$, then $S$ satisfies the $C M C$.

Under Definition 3, Theorem 7 reveals a sense in which determinism is more favorable than indeterminism for the CMC. Indeed, while I agree with Steel writing that

It is hard to see why deterministic causal systems would be more likely acyclic than indeterministic ones (Steel 2005, 16),

I will explain why deterministic systems are more likely than indeterministic ones to have non-standard CFMs satisfying JI. To that effect, let me introduce a system $S^{\prime}$ which is identical to the previously introduced $S$ except for the fact that turning the key has "an irreducible probability of .85 " of doing its job (exactly as in Steel's quantum example). $S^{\prime}$ is represented by non-standard CFM $M^{\prime}=\left(\mathbf{E}^{\prime}, q\right)$. Under previous notations, equations in $\mathbf{E}^{\prime}$ are:

$$
\begin{gathered}
V_{1}=g_{1}\left(T_{1,1}, T_{1,2}\right) \\
V_{2}=g_{2}\left(T_{2,1}, T_{2,2}\right) \\
V_{3}=g_{3}\left(T_{3,3} \times V_{1}, V_{2}, T_{3,1}, T_{3,2}\right)
\end{gathered}
$$

with $T_{3,3}$ a binary variable with possible values 0 and 1 and such that $q\left(T_{3,3}=1\right)=.85$. As a result, $M^{\prime}$ satisfies $\mathbf{J I}$ if:

$\left(1^{\prime}\right)\left\{T_{1,1}, T_{1,2}\right\},\left\{T_{2,1}, T_{2,2}\right\}$ and $\left\{T_{3,1}, T_{3,2}, T_{3,3}\right\}$ are pairwise independent;

$\left(2^{\prime}\right)\left\{T_{1,1}, T_{1,2}\right\}$ is independent from $\left\{T_{2,1}, T_{2,2}, T_{3,1}, T_{3,2}, T_{3,3}\right\}$;

$\left(3^{\prime}\right)\left\{T_{2,1}, T_{2,2}\right\}$ is independent from $\left\{T_{1,1} ; T_{1,2}, T_{3,1}, T_{3,2}, T_{3,3}\right\}$ and

$\left(4^{\prime}\right)\left\{T_{3,1}, T_{3,2}, T_{3,3}\right\}$ is independent from $\left\{T_{1,1}, T_{1,2}, T_{2,1}, T_{2,2}\right\}$.

Now suppose that all these independencies hold. Then, the independencies stated by (1) to (4) all hold. This stems from the simple probabilistic following fact: for any two sets of variables $\mathbf{Y}$ and $\mathbf{Z}$ and any variable $V$, if $\mathbf{Y} \cup\{V\}$ is independent from $\mathbf{Z}$, then $\mathbf{Y}$ is independent from $\mathbf{Z}$. Then, if $M^{\prime}$ satisfies JI, then $M$ does too. Now, the converse is not true: $\left\{T_{1,1}, T_{1,2}\right\}$ (for instance) can be independent from $\left\{T_{3,1}, T_{3,2}\right\}$ while not being independent from $\left\{T_{3,1}, T_{3,2}, T_{3,3}\right\}$.

These are no facts particular the chosen example. Indeed, the example makes clear that for any two $S$ and $S^{\prime}$ differing only by the probabilistic nature of some causal relations in $S^{\prime}$, the non-standard CFMs $M$ and $M^{\prime}$ representing them differ only by the absence from $M$ of the non-standard error terms standing for indeterminism in $M^{\prime}$. In such a case, $M$ satisfies $\mathbf{J I}$ if $M^{\prime}$ does while the converse does not hold. In this sense, non-standard CFMs representing deterministic systems are more likely than non-standard CFMs representing indeterministic systems to satisfy JI. To this exact - and acknowledgedly narrow - extent, determinism is more favorable a context than indeterminism for the truth of the CMC. 


\section{Conclusion}

Steel's claim to enlarge the usual result concerning the relationship between determinism and the CMC from the deterministic to the general case revealed unacceptable. More precisely, it was shown that Result 2 can be interpreted in terms of satisfaction of the CMC by any system with jointly independent exogenous variables only if one uses the expression "exogenous variables" in an uncommon way. Still, this way of using the expression suggested that error terms could be employed in a way more realist than the standard one. I defined the pattern of representation that stems from this suggestion, and explained how the determinism - CMC debate can take advantage of this new framework. Determinism was easily characterized in this framework and a sense in which determinism is more favorable than indeterminism for the CMC appeared: the sufficient condition we have for the truth of the CMC is such that if two systems differ only by determinism, then the deterministic one satisfies the condition whenever the indeterministic one does - and the converse does not hold.

Hinting at "non-standard causal functional models" and at Definition 3 probably constitute the most significant contribution of Steel 2005 to the debate concerning the $\mathrm{CMC}$ in general, and its relationship to determinism in particular. But this definition, as well as the sufficient condition for the truth of the CMC that was derived from it, are valid only for systems represented by causal functional models - "non-standard" ones in the case in point. Hence, this definition and this condition are useful exactly in as much as causal functional models can represent real systems, and are there interesting exactly in as much as causal functional models can represent interesting real systems. Whether they can is a difficult question that is not tackled here and would deserve more attention. 


\section{References}

Cartwright, Nancy (1999). Causal diversity and the causal Markov condition. Synthese 121(1): 3-27.

Cartwright, Nancy (2001). What is wrong with Bayes nets?. The monist 84(2): $242-264$.

Halpern, Joe, and Judea Pearl (2005). Causes and explanations: A structuralmodel approach. Part I: causes. The British journal for the philosophy of science 56(4): 843-887.

Pearl, Judea (2000). Causality. Models, reasoning, and inference. Cambridge university press.

Spirtes, Peter, Clark Glymour, and Richard Scheines (1993). Causation, prediction, and search MIT press.

Steel, Daniel (2005). Indeterminism and the causal Markov condition. The British journal for the philosophy of science 56(1): 3-26.

Williamson, Jon (2002). Learning causal relationships. Technical report 02/02, LSE, CPNSS. 


\section{Footnotes}

1. The claim is even broader, since it extends to "pseudo-indeterministic" systems. Yet these cases will not be discussed in the present paper.

2. These variables are meant to represent relevant observable aspects of the situation under consideration. Still, other variables could be considered, giving rise to different representations of one and the same system. This is not discussed in the present paper, but I will rather assume that the set of variables representing relevant observable aspects of a given system is univocally determined.

3. This example makes clear that there is no one-to-one correspondence between $\mathbf{X}$ and $\mathbf{U}$ variables of a given FM. This is an important difference between Steel's functional models and the more traditional causal models which will be considered later on.

4. Steel does not make the distinction between systems and models representing them. This is unproblematic as long as one assumes that the correspondence between systems and models is functional, which he implicitly does. Yet for reasons that will become clear later on, I will keep systems distinct from models. 